$\underline{\xi}=$ 줄

\title{
Organoids: life in three dimensions- a review
}

\author{
Akhila C.N.V ${ }^{1 *}$, Ravi Prakash A ${ }^{2}$, Rajini Kanth $\mathrm{M}^{3}$, Sreenath $\mathrm{G}^{4}$, Sowmya $\mathrm{K}^{5}$, Radhika $\mathrm{B}^{6}$ \\ ${ }^{1}$ Post Graduate Student, G. Pulla Reddy Dental College and Hospital, Kurnool \\ ${ }^{2}$ Professor and HOD, G. Pulla Reddy Dental College and Hospital, Kurnool \\ ${ }^{3}$ Professor, G. Pulla Reddy Dental College and Hospital, Kurnool \\ ${ }^{4}$ Professor, G. Pulla Reddy Dental College and Hospital, Kurnool \\ ${ }^{5}$ Post Graduate Student, G. Pulla Reddy Dental College and Hospital, Kurnool \\ ${ }^{6}$ Post Graduate Student, G. Pulla Reddy Dental College and Hospital, Kurnool \\ *Corresponding author E-mail:drakhilacnv@gmail.com
}

\begin{abstract}
Most of the diseases in humans are as a result of complex interactions occurring at cellular and molecular level. Research today has been focused in an attempt to reveal precisely the cellular evolution into pathogenesis. There are vast array of research fields, which include molecular biology, imaging techniques, etc. One of such field recently advancing worldwide is "Organotyping". It is the successor of two dimensional cell cultures. Miniature organs and disease models can be produced from cells having the ability to proliferate and differentiate, by adopting definite protocols. Organoids are the potential tools to probe human biology and diseases; thereby they may change the approach to study diseases and provide treatment, in a more beneficiary way to the patient. Also organoids are used in vaccine production, cancer research, microbiology, tissue regeneration, drug testing, etc. Clinical trials are more devastating and may cost life of patients included in study. As such, organoids can be included in the protocols of clinical trials, through which the outcome of the study can be estimated. They open the doors for newer research methods and innovations, which are in peak requirement of present day scenario where new diseases are emerging and the diseases already existing are not yet cured.
\end{abstract}

Keywords: Cell Culture in Dentistry; Organotyping; Organoids; Organoid Culture in Dentistry; Three Dimensional Cell Cultures.

\section{Introduction}

Tissue culture is a generic term used for both cell culture and organ culture. Tissue culture is widely known in producing cultures of single cell type [1]. In organ culture miniaturized replicas of organs are grown in vitro in three dimensions. Organotyping, 3D cell culture, and organoids are the names which are interchangeably used, for organ culture. Culturing tissues is a challenging task, and the applications of which outnumber its disadvantages. It is widely used in vaccine production, cancer research, microbiology, tissue regeneration, drug testing, etc. Ever since Harrison introduced method of tissue culture in 1907, the science of cell culture has been dynamic with emerging advances through years, because of its vast array of uses [2]. Over past decade the concept of organoid has revolutionized the process of tissue culture. Many organoid models are recapitulated till date, like intestinal organoids, lingual organoids, lung organoids, etc.

The pathogenesis of many diseases remains unknown as the disease process cannot be visualized and the human organs are not accessible for manipulation. Hence the concept of organoids, which are multicellular tissue proxies can fulfil the need towards understanding the disease process at cellular and molecular level and can promote research in a more focused way. Organoids obtained from cancer cells are also known to help, in the personalized treatment of patient [3].

Organoids are obtained from adult stem cells, embryonic stem cells, induced pluripotent stem cells or cancer stem cells. They are grown in vitro under certain conditions using incubator and nutrient media by maintaining the cell cycle and cell immortality. Isolation of cells for culture is done from tissue suspension by treating it with extracellular digestive enzymes like trypsin, collagenases, etc [4]. These organoid progenitor cells differentiate into tissue specific cells. Organoids are grown on a scaffold, called matrigel, which is a heterogenous gelatinous protein mixture [5]. It mimics extracellular matrix and provides environment for growth of organoids. Growth factors, organoid harvesting solution and other signaling molecules are supplied to the culture media. Imaging techniques like confocal microscopy, scanning electron microscopy and phase contrast microscopy are used to analyze organoids [6]. Protocols are established for developing various organoids, for example the procedure to develop liver organoid is different from developing salivary gland organoid [7], [8].

\section{Discussion}

Various oral structures are recapitulated by the researchers in recent years. Hisha et al cultured lingual organoid in 2013 [9]. Lingual Bmi1 positive stem cells were extracted from lingual epithelium and epidermal growth factor (EGF), Noggin, R-Spondin1 were the main 
growth factors used in their study. Spherical or budding lingual organoid was produced by them. Aihara et al (2015) and Ren et al (2014) constructed 3D model of spherical or budding taste bud organoid [10], [11]. The main growth factors used in their study were epidermal growth factor (EGF), Noggin, R-Spondin1, Jagged-1. Nanduri in 2014 in their study created a model of ductal or lobular mini-gland from salivary gland stem cells using epidermal growth factor (EGF), fibroblast growth factor-2 (FGF-2), insulin and dexamethasone [12].

\subsection{Some of the applications of organoids are as follows}

\subsubsection{Disease modelling}

Organoids are also generated from tumor biopsied tissues by directly embedding them in matrigel and help in personalized treatment for the patient [13]. Patient derived organoids are more rapidly grown when compared to other organoids. They help in screening of the active drug apt for the patient by performing drug screening assay. In 2015 a 19 year old male patient suffering with cystic fibrosis was successfully treated by this organoid culturing method [14]. Cystic fibrosis is a rare genetic disorder of lungs, kidneys and other organs and can be specifically cured with the patient derived cystic fibrosis organoid model. Researchers developed disease models of prostate cancer, pancreatic cancer, lung cancer, oral cancer, etc. [15 - 18]

\subsubsection{Drug discovery and drug screening}

Clinical trials are more devastating and may cost life of patients included in study. As such, organoids can be included in the protocols of clinical trials, through which the outcome of the study can be estimated [19]. Also a great amount of money is being spent with drug screening and development of new medicine. Despite that ninety percent of clinical trials fail while reaching phase 1 of the trial. Therefore efforts are needed to create new experimental models for drug screening, which simplifies the process. The physiology of the body can be created invitro with the integration of organoids, organ-in-chip, microfluidics, microreactors, $3 \mathrm{~d}$ bioprinting and bioink. These are the most trending terms in biotechnology. In 2017 an article was published in the Nature Scientific Reports as- "The development of an integrated platform of three organ-in-a-chip: heart, liver and lungs [20]."

\subsubsection{Gene editing}

Clustered regularly interspaced short palindromic repeats (CRISPR) and CRISPR-associated protein (Cas), is the latest advance in gene editing techniques. They are the DNA sequences of bacteria which help in altering human genome by slicing the target site and adding the desired segment of DNA. The single spheroids from organ culture allow CRISPR studies to be performed on them. Single spheroids are the intermediate stage of organoid development containing little relevant tissue [21]. They contain unaltered genetic material from parent tissue and they mimic in vivo cellular genome [22].

\subsubsection{Regenerative medicine}

Organoids can be used in regenerative medicine as tissue autologous transplants with individual's own cells, which help in minimizing graft-host immune reactions. The advantage of using organoids is that they replicate the original tissue with key features in them. For example the buccal keratinocytes cell culture can differentiate and the stratification of the cell layer along with keratin production is possible [4]. Studies are still in progress to find organoid culture technique's potential in this field.

\subsubsection{Applications in dentistry}

Oral diseases are also remodeled using this organoid technology. Like oral candidiasis, is recapitulated to observe the disease pathogenesis through candida-oral mucosal cell interactions [23]. Tumors of odontogenic origin can also be cultured to observe the cell directly involved in pathogenesis of the lesions. For example a model of ameloblastoma was created on a bone scaffold mimicking tumor-bone environment [6]. Through this, the pathogenesis, growth and gene expression are observed accurately and thereby useful for development of newer drugs and different management procedures. The toxic effects of dentifrices, oral rinsing agents, local anesthetic agents, etc. are assessed by the use of oral mucosa organoid models [4].

\section{Conclusion}

The complexity of the organoids with cell-cell interactions and differentiation make them unique from routine cell culture techniques. They serve as the potential tools, to probe human biology and diseases; thereby they change the approach to study diseases and treatment in a more beneficiary way to the patient. Further studies are required to ascertain definite protocols in developing them. Also their uses in the field of dentistry have to be explored for more sophisticated treatment methodologies.

\section{References}

[1] Jing Nie, Eri Hashino (2017) Organoid technologies meet genome engineering. EMBO Reports 18: $367-376$. https://doi.org/10.15252/embr.201643732.

[2] Eliah R. Shamir and Andrew J. Ewald (2014) Three-dimensional organotypic culture: experimental models of mammalian biology and disease. Nature Reviews Molecular Cell Biology 15: 647-664. https://doi.org/10.1038/nrm3873.

[3] Aliya et al., (2016) Organoids as an in vitro model of human development and disease. Nature Cell Biology 18: $246-254$. https://doi.org/10.1038/ncb3312.

[4] Anna and Helena (2006) Development of a highly reproducible three-dimensional organotypic model of the oral mucosa. Nature Protocols 1: 2012-2018. https://doi.org/10.1038/nprot.2006.323.

[5] Ninouk Akkerman and Libert H.K. Defize (2017) Dawn of the organoid era. Bioessays: 1600244. https://doi.org/10.1002/bies.201600244 
[6] Eriksson, Day, Fedele and Salih (2016) The regulation of bone turnover in ameloblastoma using an organotypic in vitro co-culture model. Journal of Tissue Engineering 7: $1-8$. https://doi.org/10.1177/2041731416669629.

[7] Senthil K. Muthuswamy (2017) Bringing together the organoid field: from early beginnings to the road ahead. Development 144: $963-967$. https://doi.org/10.1242/dev.144444.

[8] Takebe et al., (2017) Massive and Reproducible Production of Liver Buds Entirely from Human Pluripotent Stem Cells. Cell Reports 21: 26612670. https://doi.org/10.1016/j.celrep.2017.11.005.

[9] Hisha et al., (2016) Lingual Epithelial Stem Cells and Organoid Culture of Them. International Journal of Molecular Sciences 17: 168. https://doi.org/10.3390/ijms17020168.

[10] Aihara et al., (2015) Characterization of stem/progenitor cell cycle using murine circumvallate papilla taste bud organoid. Scientific Reports 5: 17185. https://doi.org/10.1038/srep17185.

[11] Ren W et al., (2017) Transcriptome analyses of taste organoids reveal multiple pathways involved in taste cell generation. Scientific Reports 7: 4004. https://doi.org/10.1038/s41598-017-04099-5.

[12] Nanduri et al., (2014) Purification and Ex Vivo Expansion of Fully Functional Salivary Gland Stem Cells. Stem cell reports 3: 957-964. https://doi.org/10.1016/j.stemcr.2014.09.015.

[13] Papapetrou (2016) Patient-derived induced pluripotent stem cells in cancer research and precision oncology. Nature medicine 22: $1392-1401$. https://doi.org/10.1038/nm.4238.

[14] Angela Saini (2016) Cystic Fibrosis Patients Benefit from Mini Guts. Stem Cell 6: 425-427. https://doi.org/10.1016/j.stem.2016.09.001

[15] Ma L, et al., (2017) Organoid culture of human prostate cancer cell lines LNCaP and C4-2B. American Journal of Clinical and Experimental Urology $5: 25-33$.

[16] Baker et al., (2016) Modeling pancreatic cancer with organoids. Trends in cancer 2:176-190. https://doi.org/10.1016/j.trecan.2016.03.004.

[17] Gao et al., (2017) 3D Spheroid/Organoid Models of Lung Cancer to Study Lung Cancer Pathogenesis and Testing of New Therapeutics. Journal of Thoracic Oncology 12: S1544. https://doi.org/10.1016/j.jtho.2017.06.053.

[18] Lovisa et al., (2015) Targeting Toll-like receptor 2 inhibits growth of head and neck squamous cell carcinoma. Oncotarget 6: 9897-9907. https://doi.org/10.18632/oncotarget.3393.

[19] Edmondson et al., (2014) Three-Dimensional Cell Culture Systems and Their Applications in Drug Discovery and Cell-Based Biosensors: Assay and Drug Development Technologies 12: 207-218. https://doi.org/10.1089/adt.2014.573.

[20] Skardal et al., (2017) Multi-tissue interactions in an integrated three-tissue organ-on-achip platform. Scientific reports 7: 8837. DOI: https://doi.org/10.1038/s41598-017-08879-x.

[21] Driehuis and Clevers (2017) CRISPR/Cas 9 genome editing and its applications in organoids. American Journal of Physiology 312: 257-265 https://doi.org/10.1152/ajpgi.00410.2016.

[22] Schmidt et al., (2016) Spheroid-based 3-dimensional culture models: Gene expression and functionality in head and neck cancer. Oncology Reports, 35, 2431-2440. https://doi.org/10.3892/or.2016.4581.

[23] Dongari et al., (2006) Development of a novel three-dimensional in vitro model of oral Candida infection. Microbial pathogenesis 40: 271-8. https://doi.org/10.1016/j.micpath.2006.02.004. 